\title{
A simple bijection between binary trees and colored ternary trees
}

\author{
Yidong Sun \\ Department of Mathematics, \\ Dalian Maritime University, 116026 Dalian, P.R. China \\ sydmath@yahoo.com.cn \\ Submitted: Feb 25, 2009; Accepted: Mar 28, 2010; Published: Apr 5, 2010 \\ Mathematics Subject Classification: 05C05, 05A19
}

\begin{abstract}
In this short note, we first present a simple bijection between binary trees and colored ternary trees and then derive a new identity related to generalized Catalan numbers.
\end{abstract}

Keywords: Binary tree; Ternary tree; Generalized Catalan number.

\section{Introduction}

Recently, Mansour and the author [2] obtained an identity involving 2-Catalan numbers $C_{n, 2}=\frac{1}{2 n+1}\left(\begin{array}{c}2 n+1 \\ n\end{array}\right)$ and 3-Catalan numbers $C_{n, 3}=\frac{1}{3 n+1}\left(\begin{array}{c}3 n+1 \\ n\end{array}\right)$, i.e.,

$$
\sum_{p=0}^{[n / 2]} \frac{1}{3 p+1}\left(\begin{array}{c}
3 p+1 \\
p
\end{array}\right)\left(\begin{array}{c}
n+p \\
3 p
\end{array}\right)=\frac{1}{2 n+1}\left(\begin{array}{c}
2 n+1 \\
n
\end{array}\right) .
$$

In this short note, we first present a simple bijection between complete binary trees and colored complete ternary trees and then derive the following generalized identity,

$$
\sum_{p=0}^{[n / 2]} \frac{m}{3 p+m}\left(\begin{array}{c}
3 p+m \\
p
\end{array}\right)\left(\begin{array}{c}
n+p+m-1 \\
n-2 p
\end{array}\right)=\frac{m}{2 n+m}\left(\begin{array}{c}
2 n+m \\
n
\end{array}\right) .
$$

\section{A bijective algorithm for binary and ternary trees}

A colored ternary trees is a complete ternary tree such that all its vertices are signed a nonnegative integer called color number. Let $\mathbf{T}_{n, p}$ denote the set of colored ternary trees 
$T$ with $p$ internal vertices such that the sum of all the color numbers of $T$ is $n-2 p$. Define $\mathbf{T}_{n}=\bigcup_{p=0}^{[n / 2]} \mathbf{T}_{n, p}$. Let $\mathbf{B}_{n}$ denote the set of complete binary trees with $n$ internal vertices. For any $B \in \mathbf{B}_{n}$, let $P=v_{1} v_{2} \cdots v_{k}$ be a path of length $k$ of $B$ (viewed from the root of $B$ ). $P$ is called a $R$-path, if (1) $v_{i}$ is the right child of $v_{i-1}$ for $2 \leqslant i \leqslant k$ and (2) the left child of $v_{i}$ is a leaf for $1 \leqslant i \leqslant k$. In addition, $P$ is called a maximal $R$-path if there exists no vertex $u$ such that $u P$ or $P u$ forms a $R$-path. $P$ is called an $L$-path, if $k \geqslant 2$ and $v_{i}$ is the left child of $v_{i-1}$ for $2 \leqslant i \leqslant k$. $P$ is called a maximal $L$-path if there exists no vertex $u$ such that $u P$ or $P u$ forms an $L$-path. Clearly, a leaf can never be $R$-path or $L$-path.

Note that the definition of $L$-path is different from that of $R$-path. Hence, if $P$ is a maximal $R$-path, then (1) the right child $u$ of $v_{k}$ must either be a leaf or the left child of $u$ is not a leaf; (2) $v_{1}$ must either be a left child of its father (if exists) or the father of $v_{1}$ has a left child which is not a leaf. If $P$ is a maximal $L$-path, then (1) $v_{k}$ must be a leaf which is also a left child of $v_{k-1}$; (2) $v_{1}$ must be the right child of its father (if exists).

Theorem 2.1 There exists a simple bijection $\phi$ between $\mathbf{B}_{n}$ and $\mathbf{T}_{n}$.

Proof. We first give the procedure to construct a complete binary tree from a colored complete ternary tree.

Step 1. For each vertex $v$ of $T \in \mathbf{T}_{n}$ with color number $c_{v}=k$, remove the color number and add an $R$-path $P=v_{1} v_{2} \cdots v_{k}$ of length $k$ to $v$ such that $v$ is a right child of $v_{k}$ and $v_{1}$ is a child of the father (if exists) of $v$, and then annex a left leaf to $v_{i}$ for $1 \leqslant i \leqslant k$. See Figure 1(a) for example.

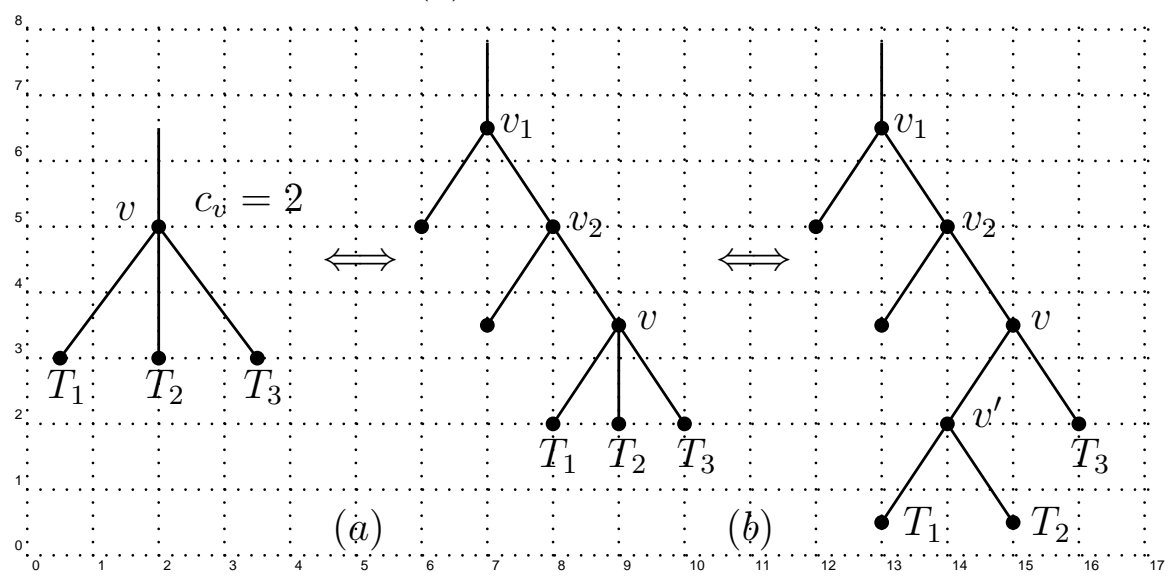

Figure 1:

Step 2. Let $T^{*}$ be the tree obtained from $T$ by Step 1 . For any internal vertex $v$ of $T^{*}$ which has out-degree 3 , let $T_{1}, T_{2}$ and $T_{3}$ be the three subtrees of $v$. Remove the subtrees $T_{1}$ and $T_{2}$, annex a left child $v^{\prime}$ to $v$ and take $T_{1}$ and $T_{2}$ as the left and right subtrees of $v^{\prime}$ respectively. See Figure 1(b) for example.

It is clear that any $T \in \mathbf{T}_{n}$, after Step 1 and 2, generates a binary tree $B \in \mathbf{B}_{n}$. Conversely, we can obtain a colored ternary tree from a complete binary tree as follows. 
Step 3. Choose any maximal $L$-path of $B \in \mathbf{B}_{n}$ of length $k$ (according to its definition, $k \geqslant 2$ ), say $P=v_{1} v_{2} \cdots v_{k}$, then each $v_{2 i-1}$ absorbs its left child $v_{2 i}$ for $1 \leqslant i \leqslant$ $[k / 2]$. This operation guarantees the resulting vertices $v_{2 i-1}$ are of out-degree 3 for $1 \leqslant i \leqslant[k / 2]$ and $v_{k}$ is always a leaf if $k$ is odd. See Figure 2(a) for example.

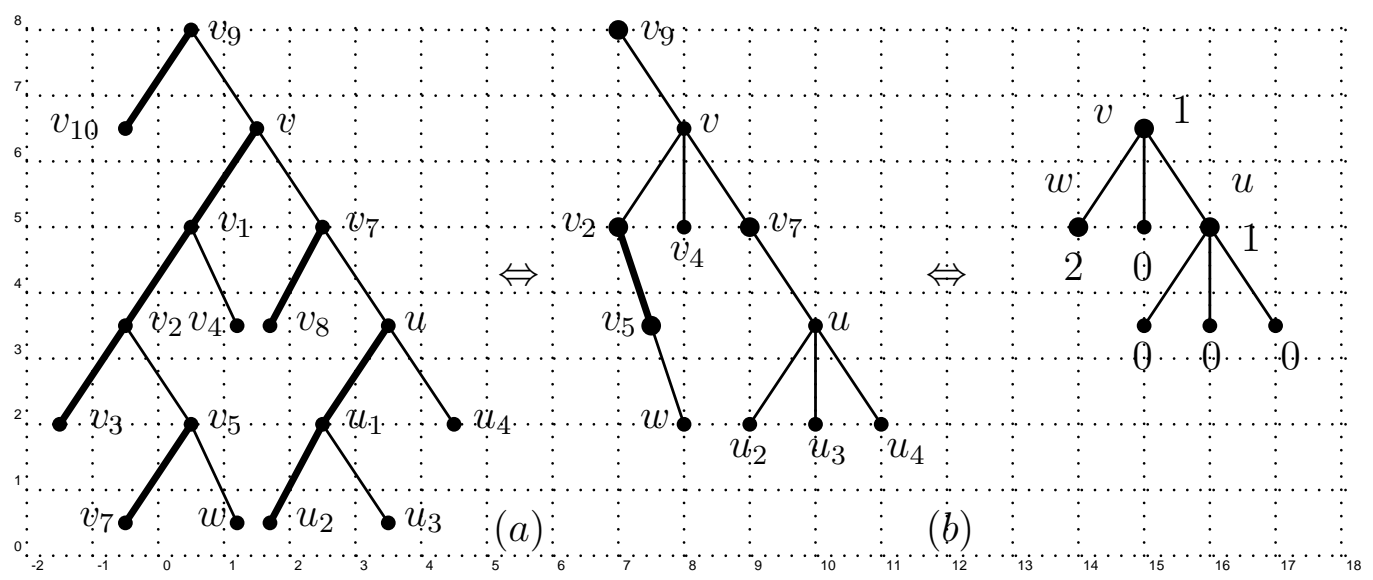

Figure 2:

Step 4. Choose any maximal $R$-path of $T^{\prime}$ derived from $B$ by Step 3 (note that any maximal $R$-path is not changed after this operation), say $Q=u_{1} u_{2} \cdots u_{k}$, let $u$ be the right child of $u_{k}$, then $u$ absorbs all the vertices $u_{1}, u_{2}, \ldots, u_{k}$ and assign the color number $c_{u}=k$ to $u$. Any remaining leaf is assigned a 0 at the end of the process. See Figure 2(b) for example. Hence we get a colored ternary tree.

Given a complete ternary tree $T$ with $p$ internal vertices, there are a total number of $3 p+1$ vertices, choose $n-2 p$ vertices with repetition allowed and define the color number of a vertex to be the number of times that vertex is chosen. Then there are $\left(\begin{array}{c}n+p \\ n-2 p\end{array}\right)$ colored ternary trees in $\mathbf{T}_{n}$ generated by $T$. Note that $\frac{1}{3 p+1}\left(\begin{array}{c}3 p+1 \\ p\end{array}\right)$ and $\frac{1}{2 n+1}\left(\begin{array}{c}2 n+1 \\ n\end{array}\right)$ count the number of complete ternary trees with $p$ internal vertices and complete binary trees with $n$ internal vertices respectively [3]. Then the bijection $\phi$ immediately leads to (1.1).

To prove (1.2), consider the forest of colored ternary trees $F=\left(T_{1}, T_{2}, \ldots, T_{m}\right)$ with $T_{i} \in \mathbf{T}_{n_{i}}$ and $n_{1}+n_{2}+\cdots+n_{m}=n$, define $\phi(F)=\left(\phi\left(T_{1}\right), \phi\left(T_{2}\right), \ldots, \phi\left(T_{m}\right)\right)$, then it is clear that $\phi$ is a bijection between forests of colored ternary trees and forests of complete binary trees. Note that there are totally $m+3 p$ vertices in a forest $F$ of complete ternary trees with $m$ components and $p$ internal vertices, so there are $\left(\begin{array}{c}m+n+p-1 \\ n-2 p\end{array}\right)$ forests of colored ternary trees with $m$ components, $p$ internal vertices and the sum of color numbers equal to $n-2 p$. It is clear from [3] that $\frac{m}{3 p+m}\left(\begin{array}{c}3 p+m \\ p\end{array}\right)$ counts the number of forests of complete ternary trees with $p$ internal vertices and $m$ components, and that $\frac{m}{2 n+m}\left(\begin{array}{c}2 n+m \\ n\end{array}\right)$ counts the number forests of complete binary trees with $n$ internal vertices and $m$ components. Then the above bijection $\phi$ immediately leads to (1.2). 
Remark: A similar type of bijection is presented by Edelman [1] in terms of noncrossing partitions.

\section{Further comments}

It is well known [3] that the $k$-Catalan number $C_{n, k}=\frac{1}{k n+1}\left(\begin{array}{c}k n+1 \\ n\end{array}\right)$ counts the number of complete $k$-ary trees with $n$ internal vertices, whose generating function $C_{k}(x)$ satisfies

$$
C_{k}(x)=1+x C_{k}(x)^{k} .
$$

Let $G(x)=\frac{1}{1-x} C_{3}\left(\frac{x^{2}}{(1-x)^{3}}\right)$, then one can deduce that

$$
\begin{aligned}
G(x) & =\frac{1}{1-x} C_{3}\left(\frac{x^{2}}{(1-x)^{3}}\right) \\
& =\frac{1}{1-x}\left(1+\frac{x^{2}}{(1-x)^{3}} C_{3}\left(\frac{x^{2}}{(1-x)^{3}}\right)^{3}\right) \\
& =\frac{1}{1-x}\left(1+x^{2} G(x)^{3}\right),
\end{aligned}
$$

which generates that $G(x)=C_{2}(x)$, the generating function for 2-Catalan numbers.

By the Lagrange inversion formula, we have

$$
\begin{aligned}
C_{3}(x)^{m} & =\sum_{p \geqslant 0} \frac{m}{3 p+m}\left(\begin{array}{c}
3 p+m \\
p
\end{array}\right) x^{p}, \\
C_{2}(x)^{m} & =\sum_{n \geqslant 0} \frac{m}{2 n+m}\left(\begin{array}{c}
2 n+m \\
n
\end{array}\right) x^{n} .
\end{aligned}
$$

Then

$$
\begin{aligned}
G(x)^{m} & =\sum_{p \geqslant 0} \frac{m}{3 p+m}\left(\begin{array}{c}
3 p+m \\
p
\end{array}\right) \frac{x^{2 p}}{(1-x)^{3 p+m}} \\
& =\sum_{n \geqslant 0} x^{n} \sum_{p=0}^{[n / 2]} \frac{m}{3 p+m}\left(\begin{array}{c}
3 p+m \\
p
\end{array}\right)\left(\begin{array}{c}
n+p+m-1 \\
n-2 p
\end{array}\right) .
\end{aligned}
$$

Comparing the coefficient of $x^{n}$ in $C_{2}(x)^{m}$ and $G(x)^{m}$, one obtains (1.2).

Similarly, let $F(x)=\frac{1}{1-x} C_{k}\left(\frac{x^{k-1}}{(1-x)^{k}}\right)$, then $F(x)=\frac{1+x F(x)}{1-x^{k-1} F(x)^{k-1}}$, using the Lagrange inversion formula for the case $k=5$, one has

$$
\begin{aligned}
& \sum_{p=0}^{[n / 4]} \frac{m}{5 p+m}\left(\begin{array}{c}
5 p+m \\
p
\end{array}\right)\left(\begin{array}{c}
n+p+m-1 \\
n-4 p
\end{array}\right) \\
& \quad=\sum_{p=0}^{[n / 2]}(-1)^{p} \frac{m}{m+n}\left(\begin{array}{c}
m+n+p-1 \\
p
\end{array}\right)\left(\begin{array}{c}
m+2 n-2 p-1 \\
n-2 p
\end{array}\right),
\end{aligned}
$$


which, in the case $m=1$, leads to

$$
\sum_{p=0}^{[n / 4]} \frac{1}{4 p+1}\left(\begin{array}{c}
5 p \\
p
\end{array}\right)\left(\begin{array}{c}
n+p \\
5 p
\end{array}\right)=\sum_{p=0}^{[n / 2]}(-1)^{p} \frac{1}{n+1}\left(\begin{array}{c}
n+p \\
n
\end{array}\right)\left(\begin{array}{c}
2 n-2 p \\
n
\end{array}\right)
$$

One may ask to give a combinatorial proof of (3.1) or (3.2). Later, based on the idea of our bijection, Yan [4] provided nice proofs for them.

\section{Acknowledgements}

The author is grateful to the anonymous referees for the helpful suggestions and comments. The work was supported by The National Science Foundation of China (Grant No. 10801020 and 70971014$)$.

\section{References}

[1] P. H. Edelman, Mutichains, non-crossing partitions and trees, Discrete Mathematics, Volume 40, (1982), 171-179.

[2] T. Mansour and Y. Sun, Bell polynomials and $k$-generalized Dyck paths, Discrete Applied Mathematics, Volume 156(12), (2008), 2279-2292.

[3] R. Stanley, Enumerative Combinatorics, vol. 2, Cambridge Univ. Press, Cambridge, 1999.

[4] S. H. F. Yan, Bijective proofs of identities from colored binary trees, The Electronic Journal of Combinatorics, Volume 15(1), (2008), \#N20. 